\title{
COMPLETE SYNCHRONIZATION OF HYPERCHAOTIC XU AND HYPERCHAOTIC LÜ SYSTEMS VIA ACTIVE CONTROL
}

\author{
Sundarapandian Vaidyanathan \\ Research and Development Centre, Vel Tech Dr. RR \& Dr. SR Technical University \\ Avadi, Chennai-600 062, Tamil Nadu, INDIA \\ sundarvtuegmail. com
}

\begin{abstract}
This paper deploys active control for achieving complete synchronization of hyperchaotic Xu (2009) and hyperchaotic Lü (2006) systems. Specifically, this paper derives complete synchronization results for identical hyperchaotic Xu systems, identical hyperchaotic Lü systems and non-identical hyperchaotic Xu and Lü systems. The complete synchronization results have been proved using Lyapunov stability theory. Numerical simulations have been shown to validate and demonstrate the effectiveness of the complete synchronization results derived in this paper.
\end{abstract}

\section{KEYWORDS}

Active Control, Synchronization, Hyperchaos, Hyperchaotic Xu System, Hyperchaotic Lü System.

\section{INTRODUCTION}

For the last few decades, chaos theory has been received critical investigations in a variety of fields including physical systems [1-2], chemical systems [3], ecological systems [4], secure communications [5-6], etc.

If we call a particular chaotic system as the master system and another chaotic system as the slave system, then the idea of complete chaos synchronization is to use the output of the master system to control the slave system so that the states of the slave system track the states of the master system asymptotically.

In the last two decades, a variety of schemes have been derived for chaos synchronization such as PC method [7], OGY method [8], active control [9-13], adaptive control [14-17], backstepping design [18-20], sampled-data feedback [21], sliding mode control [22-26], etc.

Hyperchaotic system is usually defined as a chaotic system having at least two positive Lyapunov exponents, implying that its dynamics can be extended in several directions simultaneously. Thus, hyperchaotic systems have more complex dynamical behaviour which can be used to improve the security of a chaotic communication system [27].

In this paper, we use active control method to derive new results for the complete chaos synchronization of identical hyperchaotic Xu systems ([28], 2009), identical hyperchaotic Lü systems ([29], 2006) and non-identical hyperchaotic Bao and hyperchaotic Xu systems. The complete synchronization results derived in this paper have been used using Lyapunov stability theory [30]. 


\section{Problem Statement and Our Methodology}

As the master system, we take the chaotic system described by

$$
\dot{x}=A x+f(x)
$$

where $x \in R^{n}$ is the state vector, $A$ is the $n \times n$ matrix of system parameters and $f: R^{n} \rightarrow R^{n}$ is the nonlinear part of the system.

As the slave system, we take the chaotic system described by

$$
\dot{y}=B y+g(y)+u
$$

where $y \in R^{n}$ is the state of the slave system, $B$ is the $n \times n$ matrix of system parameters, $g: R^{n} \rightarrow R^{n}$ is the nonlinear part of the system and $u$ is the active controller to be designed.

If $A=B$ and $f=g$, then $x$ and $y$ are the states of two identical chaotic systems. If $A \neq B$ or $f \neq g$, then $x$ and $y$ are the states of two different chaotic systems.

For the complete chaos synchronization of the chaotic systems (1) and (2) using active control, we define the synchronization error as

$$
e=y-x
$$

From (1), (2) and (3), the error dynamics is obtained as

$$
\dot{e}=B y-A x+g(y)-f(x)+u
$$

Thus, the complete synchronization problem is to determine a feedback controller $u$ so that

$$
\lim _{t \rightarrow \infty}\|e(t)\|=0, \text { for all } e(0) \in R^{n}
$$

Next, we consider a candidate Lyapunov function

$$
V(e)=e^{T} P e
$$

where $P$ is a positive definite matrix. Note that $V: R^{n} \rightarrow R$ is a positive definite function by construction.

If we determine a feedback controller $u$ so that

$$
\dot{V}(e)=-e^{T} Q e
$$

where $Q$ is a positive definite matrix, then $\dot{V}: R^{n} \rightarrow R$ is a negative definite function.

Thus, by Lyapunov stability theory [25], the error dynamics (4) is globally exponentially stable. Hence, the states of the master system (1) and slave system (2) are completely synchronized. 
International Journal of Computer Science \& Engineering Survey (IJCSES) Vol.3, No.3, June 2012

\section{SYSTEMS DESCRIPTION}

In this section, we give details of the hyperchaotic systems discussed in this paper.

The hyperchaotic Xu system ([28], 2009) is described by the 4D dynamics

$$
\begin{aligned}
& \dot{x}_{1}=a\left(x_{2}-x_{1}\right)+x_{4} \\
& \dot{x}_{2}=b x_{1}+\varepsilon x_{1} x_{3} \\
& \dot{x}_{3}=-c x_{3}-x_{1} x_{2} \\
& \dot{x}_{4}=x_{1} x_{3}-d x_{2}
\end{aligned}
$$

where $x_{1}, x_{2}, x_{3}, x_{4}$ are the states and $a, b, c, d, \varepsilon$ are positive constants.

The system (8) exhibits hyperchaotic behaviour when the parameter values are chosen as

$$
a=10, \quad b=40, \quad c=2.5, \quad d=2 \text { and } \varepsilon=16 .
$$

Figure 1 describes the hyperchaotic attractor of the hyperchaotic Xu system (8).
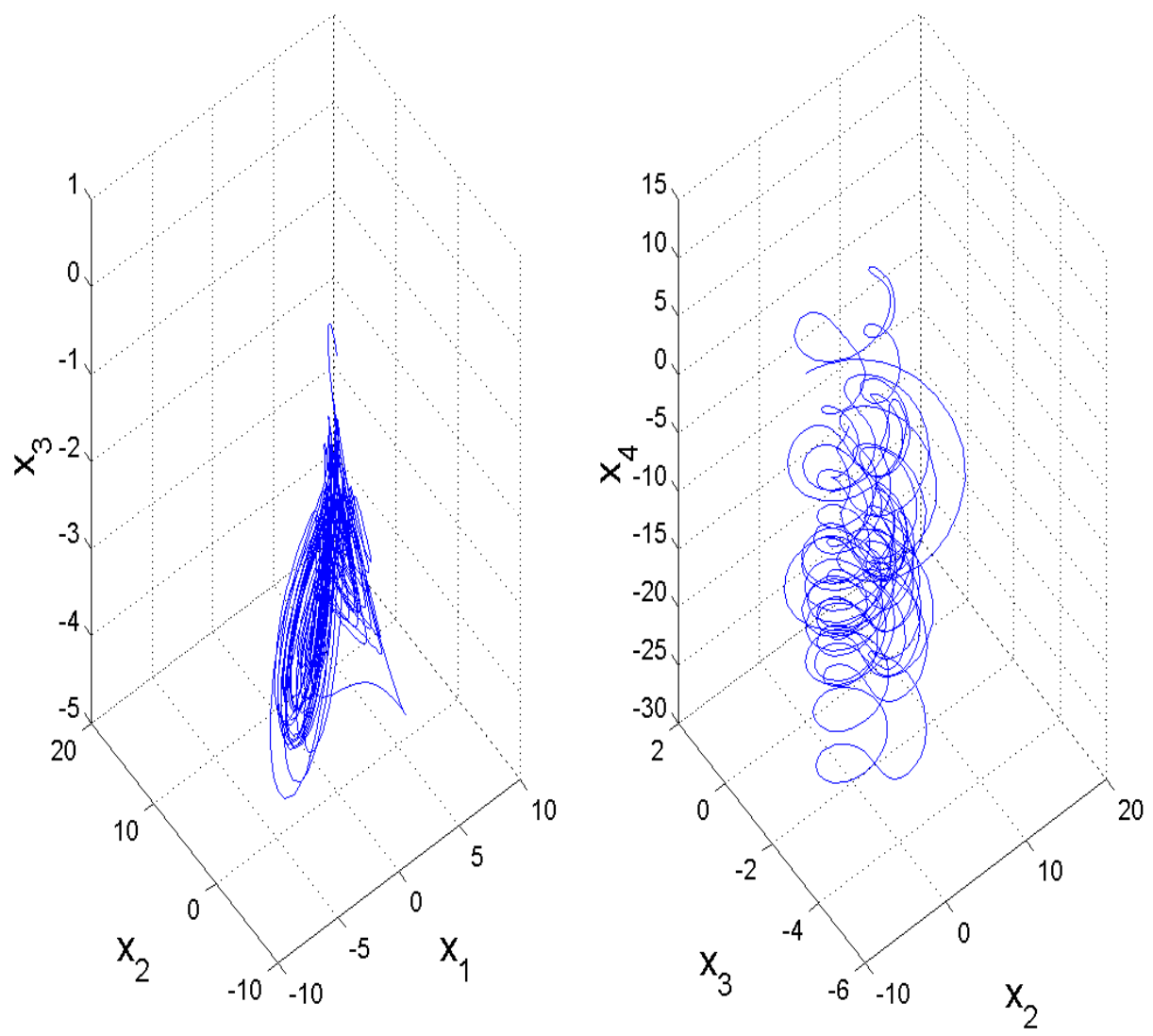

Figure 1. Hyperchaotic Attractor of the Hyperchaotic Xu System 
International Journal of Computer Science \& Engineering Survey (IJCSES) Vol.3, No.3, June 2012

The hyperchaotic Lü system ([29], 2006) is described by the 4D dynamics

$$
\begin{aligned}
& \dot{x}_{1}=\alpha\left(x_{2}-x_{1}\right)+x_{4} \\
& \dot{x}_{2}=-x_{1} x_{3}+\gamma x_{2} \\
& \dot{x}_{3}=x_{1} x_{2}-\beta x_{3} \\
& \dot{x}_{4}=x_{1} x_{3}+\delta x_{4}
\end{aligned}
$$

where $x_{1}, x_{2}, x_{3}, x_{4}$ are the states and $a, b, c, d, \varepsilon$ are positive constants.

The system (9) exhibits hyperchaotic behaviour when the parameter values are chosen as

$$
\alpha=36, \beta=3, \gamma=20 \text { and } \delta=1.3 \text {. }
$$

Figure 2 describes the hyperchaotic attractor of the hyperchaotic Lü system (9).
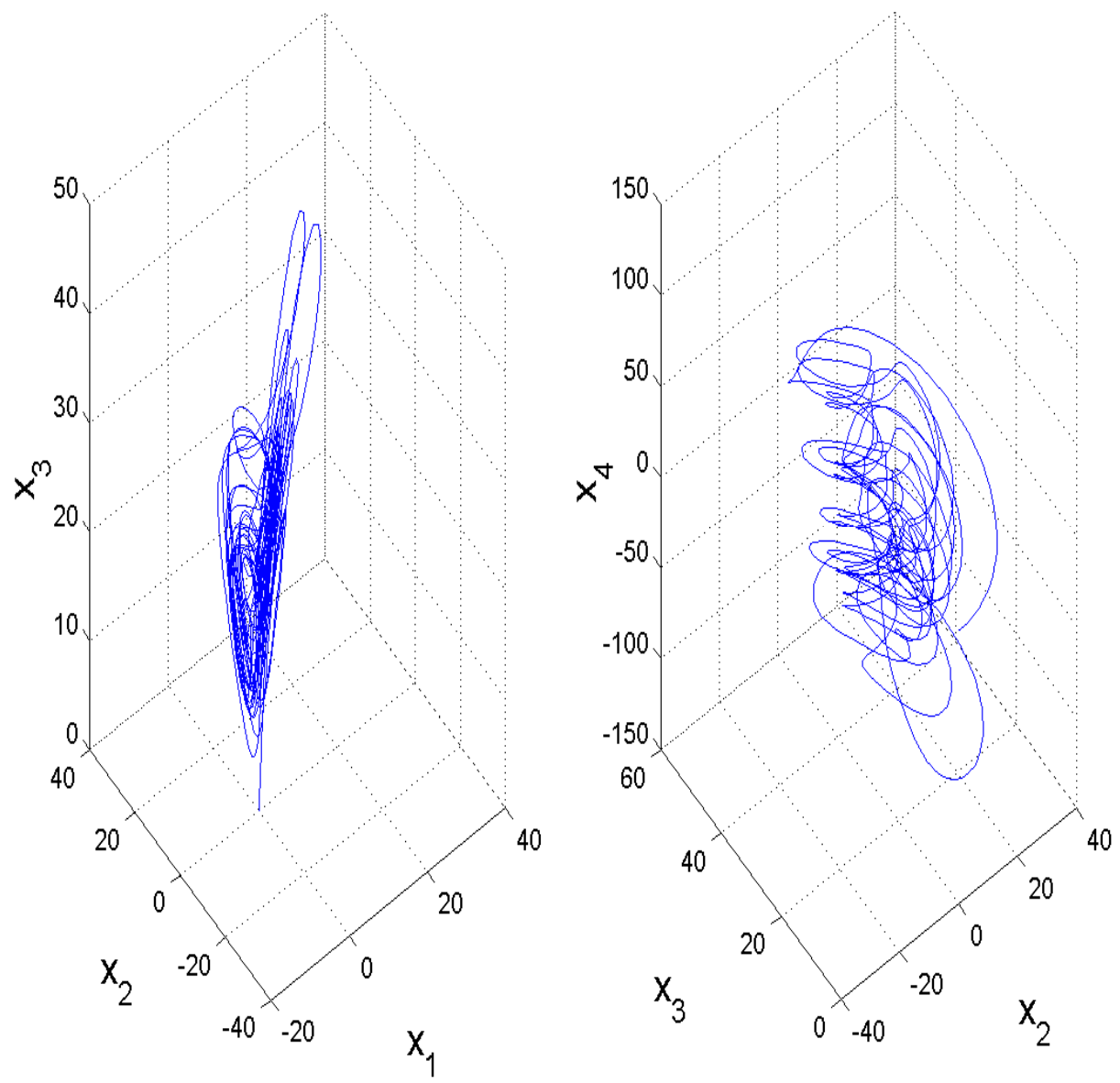

Figure 2. Hyperchaotic Attractor of the Hyperchaotic Lü System 
International Journal of Computer Science \& Engineering Survey (IJCSES) Vol.3, No.3, June 2012

\section{SyNCHRONIZATION OF IDENTICAL HyPERCHAOTIC XU SYSTEMS}

In this section, we apply active control method for the complete synchronization of identical hyperchaotic Xu systems (2009).

As the master system, we take the hyperchaotic Xu dynamics described by

$$
\begin{aligned}
& \dot{x}_{1}=a\left(x_{2}-x_{1}\right)+x_{4} \\
& \dot{x}_{2}=b x_{1}+\varepsilon x_{1} x_{3} \\
& \dot{x}_{3}=-c x_{3}-x_{1} x_{2} \\
& \dot{x}_{4}=x_{1} x_{3}-d x_{2}
\end{aligned}
$$

where $x_{1}, x_{2}, x_{3}, x_{4}$ are the state variables and $a, b, c, d, \mathcal{E}$ are positive constants.

As the slave system, we take the controlled hyperchaotic Xu dynamics described by

$$
\begin{aligned}
& \dot{y}_{1}=a\left(y_{2}-y_{1}\right)+y_{4}+u_{1} \\
& \dot{y}_{2}=b y_{1}+\varepsilon y_{1} y_{3}+u_{2} \\
& \dot{y}_{3}=-c y_{3}-y_{1} y_{2}+u_{3} \\
& \dot{y}_{4}=y_{1} y_{3}-d y_{2}+u_{4}
\end{aligned}
$$

where $y_{1}, y_{2}, y_{3}, y_{4}$ are the state variables and $u_{1}, u_{2}, u_{3}, u_{4}$ are the active controls.

The synchronization error is defined as

$$
e_{1}=y_{1}-x_{1}, e_{2}=y_{2}-x_{2}, e_{3}=y_{3}-x_{3}, e_{4}=y_{4}-x_{4}
$$

A simple calculation gives the error dynamics

$$
\begin{aligned}
& \dot{e}_{1}=a\left(e_{2}-e_{1}\right)+e_{4}+u_{1} \\
& \dot{e}_{2}=b e_{1}+\varepsilon\left(y_{1} y_{3}-x_{1} x_{3}\right)+u_{2} \\
& \dot{e}_{3}=-c e_{3}-y_{1} y_{2}+x_{1} x_{2}+u_{3} \\
& \dot{e}_{4}=y_{1} y_{3}-x_{1} x_{3}-d e_{2}+u_{4}
\end{aligned}
$$

We consider the active nonlinear controller defined by

$$
\begin{aligned}
& u_{1}=-a\left(e_{2}-e_{1}\right)-e_{4}-k_{1} e_{1} \\
& u_{2}=-b e_{1}-\varepsilon\left(y_{1} y_{3}-x_{1} x_{3}\right)-k_{2} e_{2} \\
& u_{3}=c e_{3}+y_{1} y_{2}-x_{1} x_{2}-k_{3} e_{3} \\
& u_{4}=-y_{1} y_{3}+x_{1} x_{3}+d e_{2}-k_{4} e_{4}
\end{aligned}
$$

where the gains $k_{1}, k_{2}, k_{3}, k_{4}$ are positive constants. 
International Journal of Computer Science \& Engineering Survey (IJCSES) Vol.3, No.3, June 2012

Substitution of (14) into (13) yields the linear error dynamics

$$
\dot{e}_{1}=-k_{1} e_{1}, \quad \dot{e}_{2}=-k_{2} e_{2}, \quad \dot{e}_{3}=-k_{3} e_{3}, \dot{e}_{4}=-k_{4} e_{4}
$$

Theorem 4.1. The identical hyperchaotic Xu systems (10) and (11) are globally and exponentially synchronized with the active nonlinear controller (14), where the gains $k_{i},(i=1,2,3,4)$ are positive constants.

Proof. Consider the quadratic Lyapunov function defined by

$$
V(e)=\frac{1}{2} e^{T} e=\frac{1}{2}\left(e_{1}^{2}+e_{2}^{2}+e_{3}^{2}+e_{4}^{2}\right)
$$

which is a positive definite function on $R^{4}$.

Differentiating (16) along the trajectories of the error system (15), we get

$$
\dot{V}(e)=-k_{1} e_{1}^{2}-k_{2} e_{2}^{2}-k_{3} e_{3}^{2}-k_{4} e_{4}^{2},
$$

which is a negative definite function on $R^{4}$ since $k_{1}, k_{2}, k_{3}, k_{4}$ are positive constants.

Thus, by Lyapunov stability theory [30], the error dynamics (15) is globally exponentially stable.

\section{Numerical Simulations}

For the numerical simulations, the fourth order Runge-Kutta method with initial timestep $h=10^{-8}$ is used to solve the two systems (10) and (11) with the active controller (14). We take the gains as $k_{i}=5$ for $i=1,2,3,4$.

The parameters of the identical hyperchaotic Xu systems (10) and (11) are selected as

$$
a=10, \quad b=40, c=2.5, d=2 \quad \text { and } \quad \varepsilon=16 .
$$

The initial values for the master system (10) are taken as

$$
x_{1}(0)=5, \quad x_{2}(0)=-6, \quad x_{3}(0)=12, \quad x_{4}(0)=-15
$$

and the initial values for the slave system (11) are taken as

$$
y_{1}(0)=-14, \quad y_{2}(0)=8, \quad y_{3}(0)=18, \quad y_{4}(0)=16
$$

Figure 3 shows the complete synchronization of the identical hyperchaotic Xu systems.

Figure 4 shows the time-history of the synchronization error $e_{1}, e_{2}, e_{3}, e_{4}$. 
International Journal of Computer Science \& Engineering Survey (IJCSES) Vol.3, No.3, June 2012
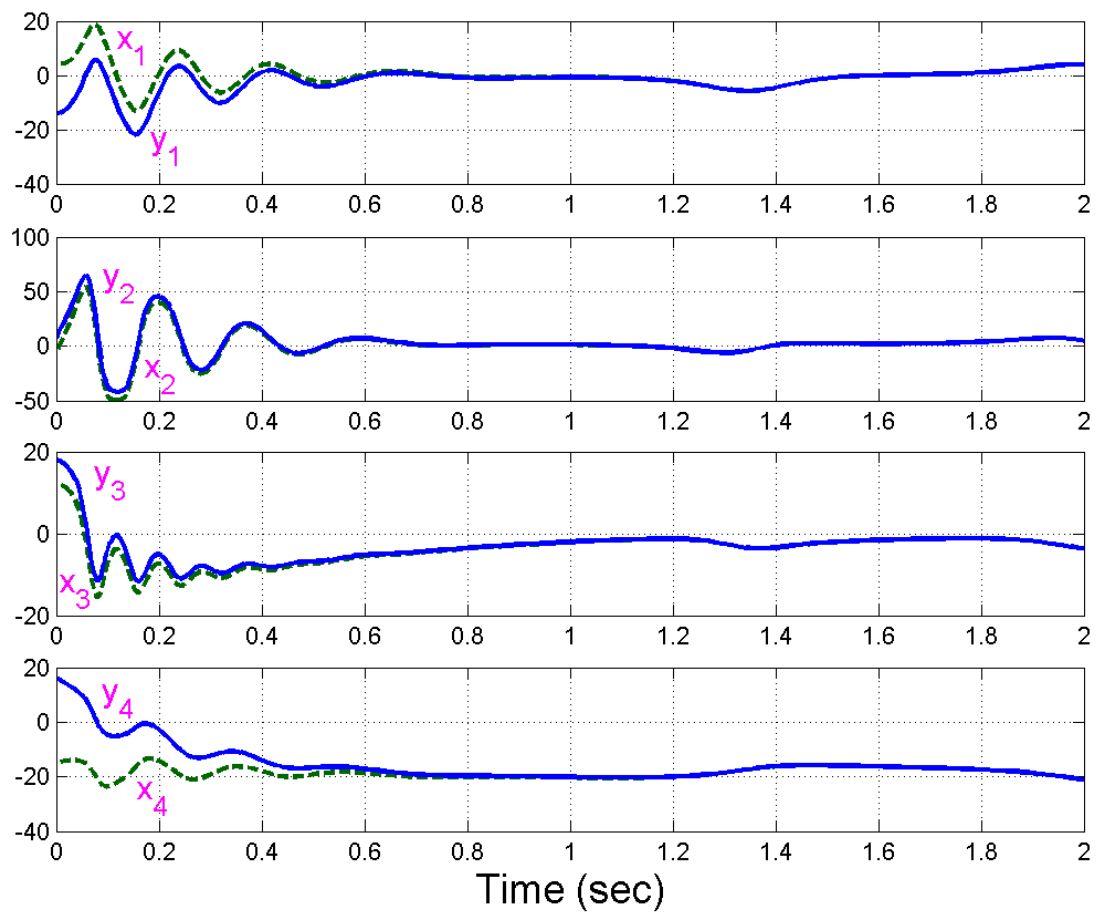

Figure 3. Synchronization of Identical Hyperchaotic Xu Systems

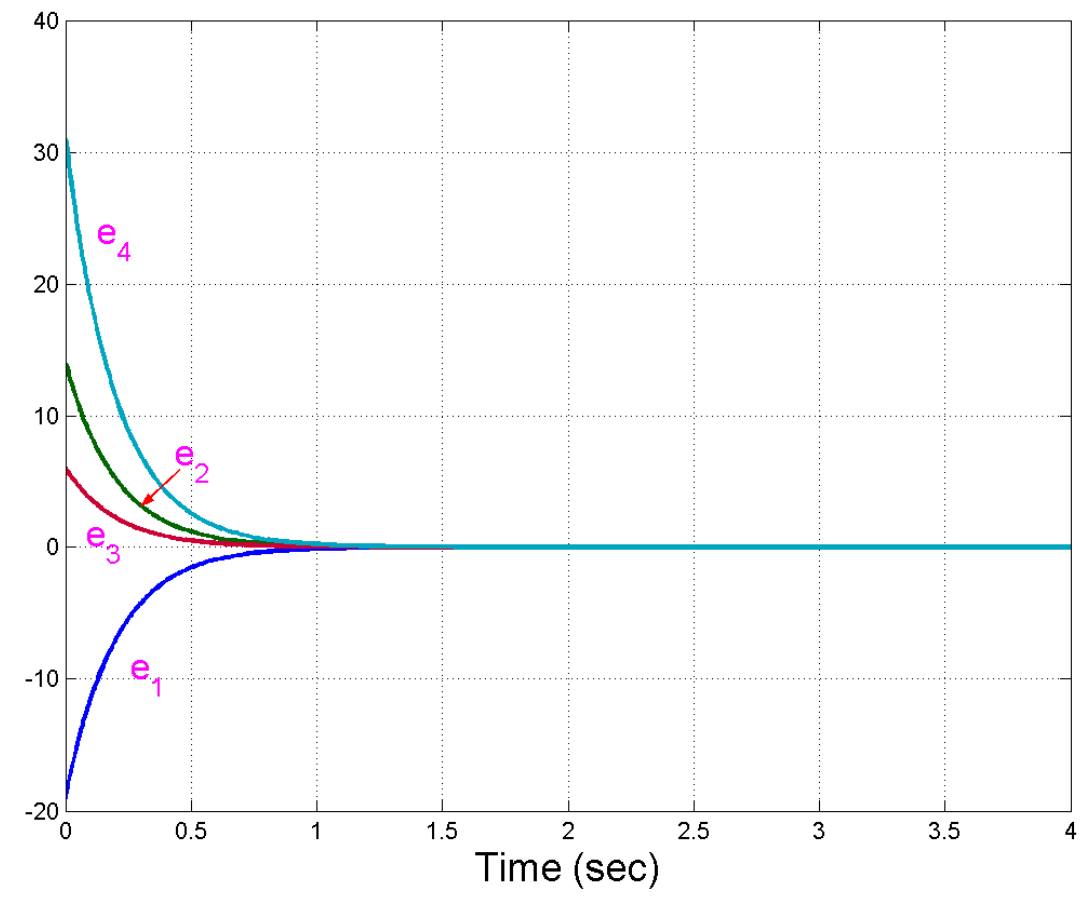

Figure 4. Time History of the Synchronization Error $e_{1}, e_{2}, e_{3}, e_{4}$ 
International Journal of Computer Science \& Engineering Survey (IJCSES) Vol.3, No.3, June 2012

\section{SYNCHRONIZATION OF IDENTICAL HyPERCHAOTIC LÜ SYSTEMS}

In this section, we apply active control method for the complete synchronization of identical hyperchaotic Lü systems (2006).

As the master system, we take the hyperchaotic Lü dynamics described by

$$
\begin{aligned}
& \dot{x}_{1}=\alpha\left(x_{2}-x_{1}\right)+x_{4} \\
& \dot{x}_{2}=-x_{1} x_{3}+\gamma x_{2} \\
& \dot{x}_{3}=x_{1} x_{2}-\beta x_{3} \\
& \dot{x}_{4}=x_{1} x_{3}+\delta x_{4}
\end{aligned}
$$

where $x_{1}, x_{2}, x_{3}, x_{4}$ are the state variables and $\alpha, \beta, \gamma, \delta$ are positive constants.

As the slave system, we take the controlled hyperchaotic Lü dynamics described by

$$
\begin{aligned}
& \dot{y}_{1}=\alpha\left(y_{2}-y_{1}\right)+y_{4}+u_{1} \\
& \dot{y}_{2}=-y_{1} y_{3}+\gamma y_{2}+u_{2} \\
& \dot{y}_{3}=y_{1} y_{2}-\beta y_{3}+u_{3} \\
& \dot{y}_{4}=y_{1} y_{3}+\delta y_{4}+u_{4}
\end{aligned}
$$

where $y_{1}, y_{2}, y_{3}, y_{4}$ are the state variables and $u_{1}, u_{2}, u_{3}, u_{4}$ are the active controls.

The synchronization error is defined as

$$
e_{1}=y_{1}-x_{1}, e_{2}=y_{2}-x_{2}, e_{3}=y_{3}-x_{3}, e_{4}=y_{4}-x_{4}
$$

A simple calculation gives the error dynamics

$$
\begin{aligned}
& \dot{e}_{1}=\alpha\left(e_{2}-e_{1}\right)+e_{4}+u_{1} \\
& \dot{e}_{2}=\gamma e_{2}+y_{1} y_{3}-x_{1} x_{3}+u_{2} \\
& \dot{e}_{3}=-\beta e_{3}-y_{1} y_{2}+x_{1} x_{2}+u_{3} \\
& \dot{e}_{4}=\delta e_{4}+y_{1} y_{3}-x_{1} x_{3}+u_{4}
\end{aligned}
$$

We consider the active nonlinear controller defined by

$$
\begin{aligned}
& u_{1}=-\alpha\left(e_{2}-e_{1}\right)-e_{4}-k_{1} e_{1} \\
& u_{2}=-\gamma e_{2}+y_{1} y_{3}-x_{1} x_{3}-k_{2} e_{2} \\
& u_{3}=\beta e_{3}-y_{1} y_{2}+x_{1} x_{2}-k_{3} e_{3} \\
& u_{4}=-\delta e_{4}-y_{1} y_{3}+x_{1} x_{3}+d e_{2}-k_{4} e_{4}
\end{aligned}
$$

where the gains $k_{1}, k_{2}, k_{3}, k_{4}$ are positive constants. 
International Journal of Computer Science \& Engineering Survey (IJCSES) Vol.3, No.3, June 2012

Substitution of (22) into (21) yields the linear error dynamics

$$
\dot{e}_{1}=-k_{1} e_{1}, \quad \dot{e}_{2}=-k_{2} e_{2}, \quad \dot{e}_{3}=-k_{3} e_{3}, \dot{e}_{4}=-k_{4} e_{4}
$$

Theorem 5.1. The identical hyperchaotic Lü systems (18) and (19) are globally and exponentially synchronized with the active nonlinear controller (22), where the gains $k_{i},(i=1,2,3,4)$ are positive constants.

Proof. Consider the quadratic Lyapunov function defined by

$$
V(e)=\frac{1}{2} e^{T} e=\frac{1}{2}\left(e_{1}^{2}+e_{2}^{2}+e_{3}^{2}+e_{4}^{2}\right)
$$

which is a positive definite function on $R^{4}$.

Differentiating (24) along the trajectories of the error system (23), we get

$$
\dot{V}(e)=-k_{1} e_{1}^{2}-k_{2} e_{2}^{2}-k_{3} e_{3}^{2}-k_{4} e_{4}^{2},
$$

which is a negative definite function on $R^{4}$ since $k_{1}, k_{2}, k_{3}, k_{4}$ are positive constants.

Thus, by Lyapunov stability theory [30], the error dynamics (23) is globally exponentially stable.

\section{Numerical Simulations}

For the numerical simulations, the fourth order Runge-Kutta method with initial timestep $h=10^{-8}$ is used to solve the two systems (18) and (19) with the active controller (22). We take the gains as $k_{i}=5$ for $i=1,2,3,4$.

The parameters of the identical hyperchaotic Lü systems (18) and (19) are selected as

$$
\alpha=36, \quad \beta=3, \gamma=20 \text { and } \delta=1.3 \text {. }
$$

The initial values for the master system (18) are taken as

$$
x_{1}(0)=8, x_{2}(0)=26, x_{3}(0)=-15, x_{4}(0)=-9
$$

and the initial values for the slave system (19) are taken as

$$
y_{1}(0)=-4, \quad y_{2}(0)=18, \quad y_{3}(0)=-8, \quad y_{4}(0)=19
$$

Figure 5 shows the complete synchronization of the identical hyperchaotic Lü systems.

Figure 6 shows the time-history of the synchronization error $e_{1}, e_{2}, e_{3}, e_{4}$. 
International Journal of Computer Science \& Engineering Survey (IJCSES) Vol.3, No.3, June 2012
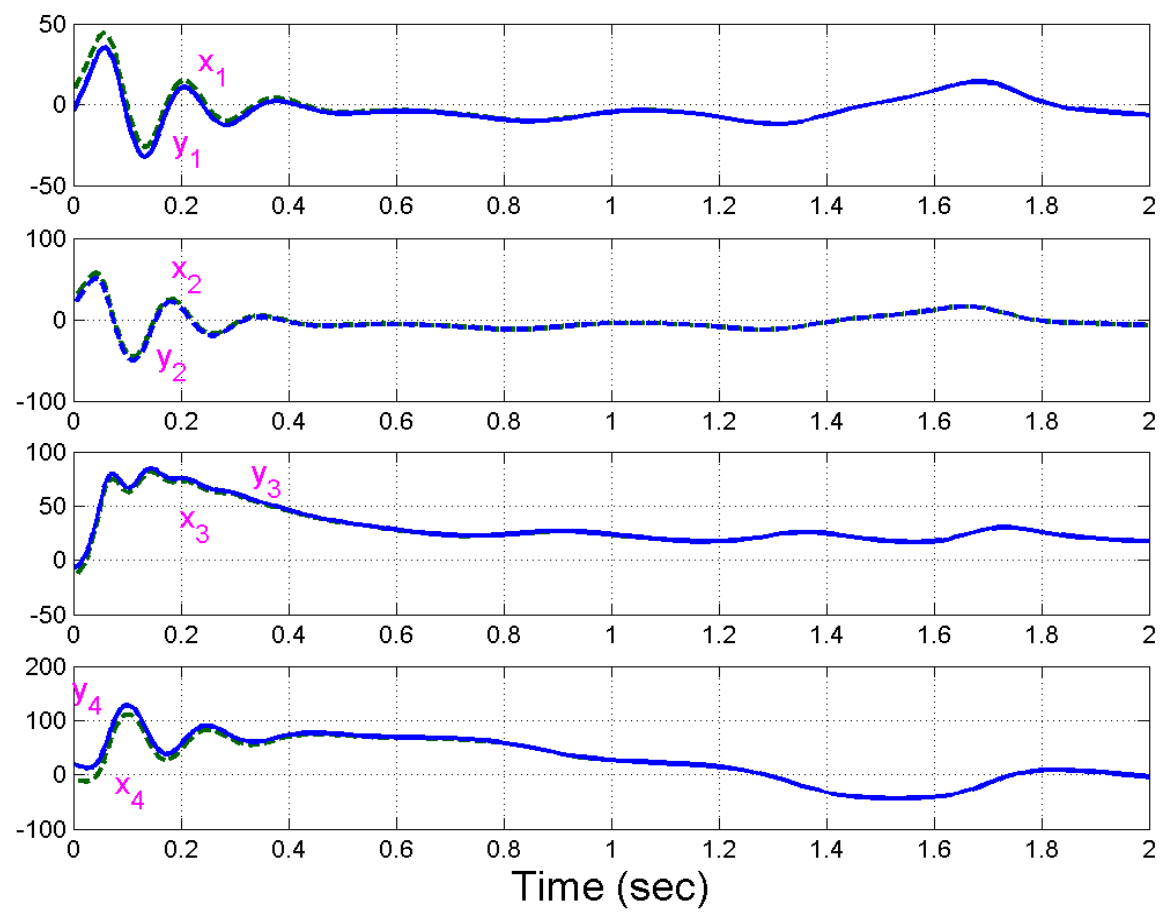

Figure 5. Synchronization of Identical Hyperchaotic Lü Systems

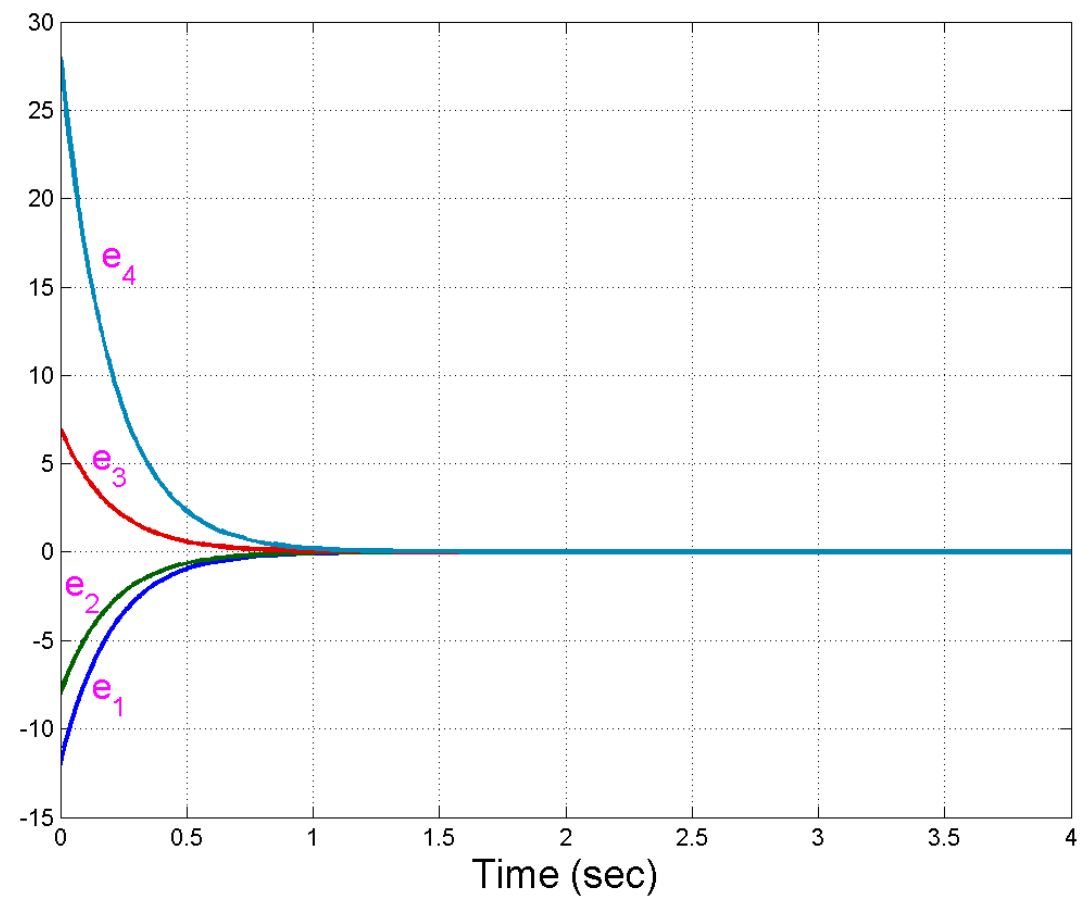

Figure 6. Time History of the Synchronization Error $e_{1}, e_{2}, e_{3}, e_{4}$ 
International Journal of Computer Science \& Engineering Survey (IJCSES) Vol.3, No.3, June 2012

\section{Synchronization of Non-IDEnTical Hyperchaotic Xu AND HYPERCHAOTIC LÜ SYSTEMS}

In this section, we consider the global chaos synchronization of non-identical hyperchaotic Xu system ([28], 2009) and hyperchaotic Lü system ([29], 2006).

As the master system, we take the hyperchaotic Xu dynamics described by

$$
\begin{aligned}
& \dot{x}_{1}=a\left(x_{2}-x_{1}\right)+x_{4} \\
& \dot{x}_{2}=b x_{1}+\varepsilon x_{1} x_{3} \\
& \dot{x}_{3}=-c x_{3}-x_{1} x_{2} \\
& \dot{x}_{4}=x_{1} x_{3}-d x_{2}
\end{aligned}
$$

where $x_{1}, x_{2}, x_{3}, x_{4}$ are the state variables and $a, b, c, d$ are positive constants.

As the slave system, we take the controlled hyperchaotic Lü dynamics described by

$$
\begin{aligned}
& \dot{y}_{1}=\alpha\left(y_{2}-y_{1}\right)+y_{4}+u_{1} \\
& \dot{y}_{2}=-y_{1} y_{3}+\gamma y_{2}+u_{2} \\
& \dot{y}_{3}=y_{1} y_{2}-\beta y_{3}+u_{3} \\
& \dot{y}_{4}=y_{1} y_{3}+\delta y_{4}+u_{4}
\end{aligned}
$$

where $y_{1}, y_{2}, y_{3}, y_{4}$ are the state variables, $\alpha, \beta, \gamma, \delta$ are positive constants and $u_{1}, u_{2}, u_{3}, u_{4}$ are the active controls.

The synchronization error is defined as

$$
e_{1}=y_{1}-x_{1}, e_{2}=y_{2}-x_{2}, e_{3}=y_{3}-x_{3}, e_{4}=y_{4}-x_{4}
$$

A simple calculation gives the error dynamics

$$
\begin{aligned}
& \dot{e}_{1}=\alpha\left(e_{2}-e_{1}\right)+(\alpha-a)\left(x_{2}-x_{1}\right)-e_{4}+u_{1} \\
& \dot{e}_{2}=\gamma y_{2}-b x_{1}-y_{1} y_{3}-\varepsilon x_{1} x_{3}+u_{2} \\
& \dot{e}_{3}=-\beta e_{3}+(c-\beta) x_{3}+y_{1} y_{2}+x_{1} x_{2}+u_{3} \\
& \dot{e}_{4}=\delta y_{4}+d x_{2}+y_{1} y_{3}-x_{1} x_{3}+u_{4}
\end{aligned}
$$

We consider the active nonlinear controller defined by

$$
\begin{aligned}
& u_{1}=-\alpha\left(e_{2}-e_{1}\right)-(\alpha-a)\left(x_{2}-x_{1}\right)+e_{4}-k_{1} e_{1} \\
& u_{2}=-\gamma y_{2}+b x_{1}+y_{1} y_{3}+\varepsilon x_{1} x_{3}-k_{2} e_{2} \\
& u_{3}=\beta e_{3}-(c-\beta) x_{3}-y_{1} y_{2}-x_{1} x_{2}-k_{3} e_{3} \\
& u_{4}=-\delta y_{4}-d x_{2}-y_{1} y_{3}+x_{1} x_{3}-k_{4} e_{4}
\end{aligned}
$$

where the gains $k_{1}, k_{2}, k_{3}, k_{4}$ are positive constants. 
International Journal of Computer Science \& Engineering Survey (IJCSES) Vol.3, No.3, June 2012

Substitution of (30) into (29) yields the linear error dynamics

$$
\dot{e}_{1}=-k_{1} e_{1}, \quad \dot{e}_{2}=-k_{2} e_{2}, \quad \dot{e}_{3}=-k_{3} e_{3}, \dot{e}_{4}=-k_{4} e_{4}
$$

Theorem 6.1. The hyperchaotic Xu system (26) and hyperchaotic Lü system (26) are globally and exponentially synchronized with the active nonlinear controller (30), where the gains $k_{i},(i=1,2,3,4)$ are positive constants.

Proof. Consider the quadratic Lyapunov function defined by

$$
V(e)=\frac{1}{2} e^{T} e=\frac{1}{2}\left(e_{1}^{2}+e_{2}^{2}+e_{3}^{2}+e_{4}^{2}\right)
$$

which is a positive definite function on $R^{4}$.

Differentiating (24) along the trajectories of the error system (23), we get

$$
\dot{V}(e)=-k_{1} e_{1}^{2}-k_{2} e_{2}^{2}-k_{3} e_{3}^{2}-k_{4} e_{4}^{2},
$$

which is a negative definite function on $R^{4}$ since $k_{1}, k_{2}, k_{3}, k_{4}$ are positive constants.

Thus, by Lyapunov stability theory [30], the error dynamics (31) is globally exponentially stable.

\section{Numerical Simulations}

For the numerical simulations, the fourth order Runge-Kutta method with initial timestep $h=10^{-8}$ is used to solve the two systems (26) and (27) with the active controller (22). We take the gains as $k_{i}=5$ for $i=1,2,3,4$.

The parameters of the hyperchaotic Xu and hyperchaotic Lü systems are selected as

$$
a=10, b=40, c=2.5, d=2, \varepsilon=16, \alpha=36, \beta=3, \gamma=20, \delta=1.3
$$

The initial values for the master system (26) are taken as

$$
x_{1}(0)=-12, \quad x_{2}(0)=16, \quad x_{3}(0)=25, \quad x_{4}(0)=-19
$$

and the initial values for the slave system (27) are taken as

$$
y_{1}(0)=24, \quad y_{2}(0)=8, \quad y_{3}(0)=-14, \quad y_{4}(0)=6
$$

Figure 7 shows the complete synchronization of the hyperchaotic $\mathrm{Xu}$ and hyperchaotic Lü systems.

Figure 8 shows the time-history of the synchronization error $e_{1}, e_{2}, e_{3}, e_{4}$. 
International Journal of Computer Science \& Engineering Survey (IJCSES) Vol.3, No.3, June 2012
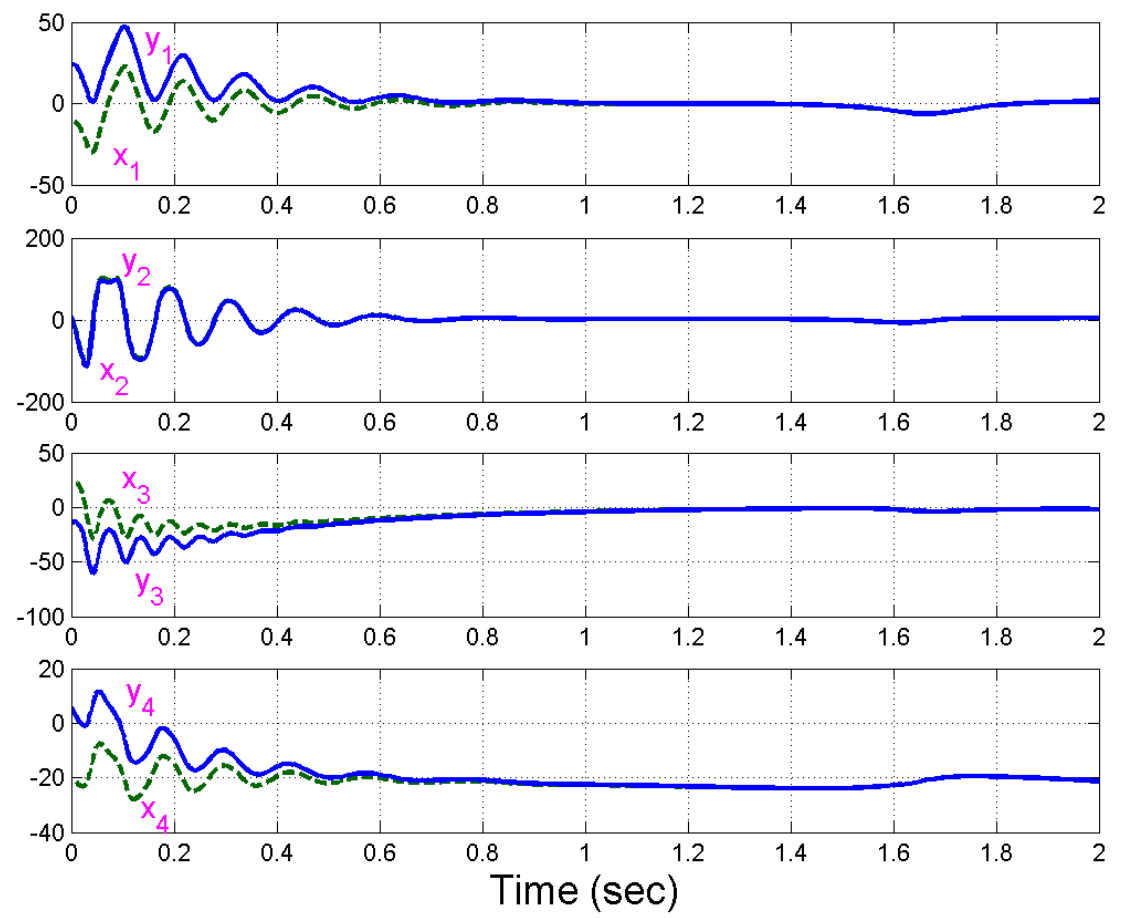

Figure 7. Synchronization of Hyperchaotic Xu and Hyperchaotic Lü Systems

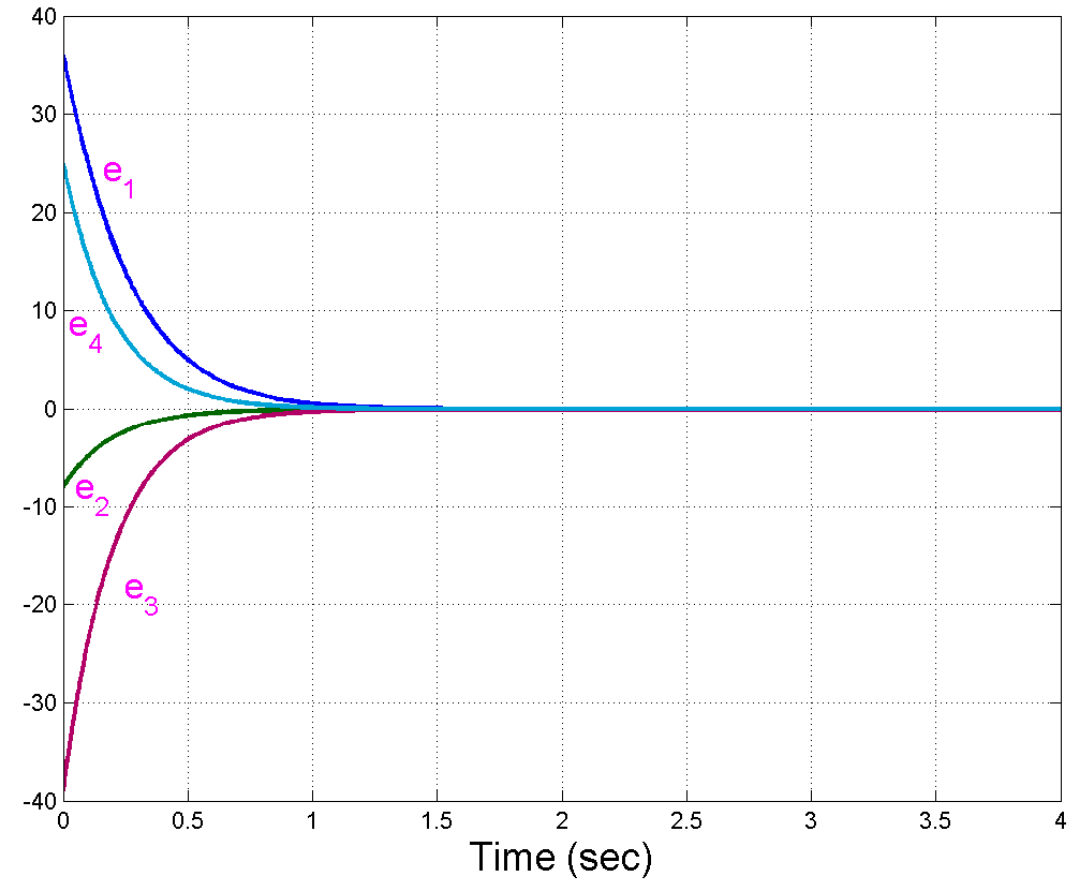

Figure 8. Time History of the Synchronization Error $e_{1}, e_{2}, e_{3}, e_{4}$ 


\section{CONClusions}

Using the active control method, we have derived new results for the complete synchronization of the identical hyperchaotic Xu systems (2009), identical hyperchaotic Lü systems (2006) and non-identical hyperchaotic Xu and hyperchaotic Lü systems. The complete synchronization results derived in this paper have been proved using Lyapunov stability theory. Numerical simulation results have been shown to demonstrate the complete synchronization results derived in this paper.

\section{REFERENCES}

[1] Lorenz, E.N. (1963) “Deterministic nonperiodic flow”, J. Atmos. Sci., Vol. 20, pp 130-141.

[2] Lakshmanan, M. \& Murali, K. (1996) Nonlinear Oscillators: Controlling and Synchronization, World Scientific, Singapore.

[3] Han, S.K., Kerrer, C. \& Kuramoto, Y. (1995) "Dephasing and burstling in coupled neural oscillators", Phys. Rev. Lett., Vol. 75, pp 3190-3193.

[4] Blasius, B., Huppert, A. \& Stone, L. (1999) "Complex dynamics and phase synchronization in spatially extended ecological system”, Nature, Vol. 399, pp 354-359.

[5] Feki, M. (2003) "An adaptive chaos synchronization scheme applied to secure communication", Chaos, Solitons and Fractals, Vol. 18, pp 141-148.

[6] Murali, K. \& Lakshmanan, M. (1998) "Secure communication using a compound signal from generalized synchronizable chaotic systems”, Phys. Rev. Lett. A, Vol. 241, pp 303-310.

[7] Pecora, L.M. \& Carroll, T.L. (1990) "Synchronization in chaotic systems", Phys. Rev. Lett., Vol. 64, pp 821-824.

[8] Ott, E., Grebogi, C. \& Yorke, J.A. (1990) "Controlling chaos”, Phys. Rev. Lett., Vol. 64, pp 1196-1199.

[9] Ho, M.C. \& Hung, Y.C. (2002) "Synchronization of two different chaotic systems by using generalized active control", Physics Letters A, Vol. 301, pp. 424-428.

[10] Chen, H.K. (2005) "Global chaos synchronization of new chaotic systems via nonlinear control”, Chaos, Solitons \& Fractals, Vol. 23, pp. 1245-1251.

[11] Sundarapandian, V. \& Rasappan, S. (2010) "Global chaos synchronization of Newton-Leipnik system and Liu-Chen four scroll chaotic attractor by nonlinear control," International Journal of Control Theory and Applications, Vol. 3, No. 1, pp 29-36.

[12] Sundarapandian, V. (2011) "Global chaos synchronization of four-scroll and four-wing chaotic attractors by active nonlinear control," International Journal on Computer Science and Engineering, Vol. 3, No. 5, pp 2145-2155.

[13] Sundarapandian, V. (2011) "Anti-synchronization of Arneodo and Coullet systems by active nonlinear control," International Journal of Control Theory and Applications, Vol. 4, No. 1, pp 25-36.

[14] Liao, T.L. \& Tsai, S.H. (2000) “Adaptive synchronization of chaotic systems and its applications to secure communications”, Chaos, Solitons and Fractals, Vol. 11, pp 1387-1396.

[15] Sundarapandian, V. (2011) “Adaptive control and synchronization of hyperchaotic Cai system”, International Journal of Control Theory and Computer Modelling, Vol. 1, No. 1, pp. 1-13.

[16] Sundarapandian, V. (2011) "Adaptive synchronization of hyperchaotic Lorenz and hyperchaotic Liu systems”, International Journal of Instrumentation and Control Systems, Vol. 1, No. 1, pp. $1-18$.

[17] Sundarapandian, V. (2011) "Adaptive control and synchronization of a highly chaotic attractor," International Journal of Information Sciences and Techniques, Vol. 1, No. 2, pp 1-11. 
[18] Tan, X., Zhang, J. \& Yang, Y. (2003) "Synchronizing chaotic systems using backstepping design," Chaos, Solitons \& Fractals, Vol. 16, pp 37-45.

[19] Yu, Y.G. \& Zhang, S.C. (2006) “Adaptive backstepping synchronization of uncertain chaotic systems”, Chaos, Solitons \& Fractals, Vol. 27, pp 1369-1375.

[20] Laoye, J.A., Vincent, U.E. \& Kareem, S.O. (2009) "Chaos control of 4-D chaotic system using recursive backstepping nonlinear controller," Chaos, Solitons \& Fractals, Vol. 39, pp 356-362.

[21] Yang, T. \& Chua, L.O. (1999) “Control of chaos using sampled-data feedback control”, Internat. J. Bifurcat. Chaos, Vol. 9, pp 215-219.

[22] Sundarapandian, V. (2011) "Global chaos synchronization of four-wing chaotic systems by sliding mode control”, International Journal of Control Theory and Computer Modelling, Vol. 1, No. 1, pp. 15-31.

[23] Sundarapandian, V. (2011) "Global chaos synchronization of Pehlivan systems by sliding mode control”, International Journal on Computer Science and Engineering, Vol. 3, No. 5, pp. 21632169.

[24] Sundarapandian, V. (2011) "Sliding mode controller design for the synchronization of ShimizuMorioka chaotic systems”, International Journal of Information Sciences and Techniques, Vol. 1, No. 1, pp 20-29.

[25] Sundarapandian, V. (2011) "Hybrid synchronization of hyperchaotic Newton-Leipnik systems via sliding mode control," International Journal of Control Theory and Computer Modelling, Vol. 1, No. 2, pp 1-10.

[26] Sundarapandian, V. (2012) "Anti-synchronization of Pan systems via sliding mode control," International Journal of Information Technology, Control and Automation, Vol. 2, No. 2, pp 1525 .

[27] Chen, S.L., Chang, S.M., Lin, W.W. \& Hwang, T. (2008) "Digital secure communication using robust hyperchaotic systems," International Journal of Bifurcation and Chaos, Vol. 18, No. 11, pp 3325-3339.

[28] Xu, J., Cai, G. \& Zheng, S. (2009) "A novel hyperchaotic system and its control”, J. Uncertain Systems, Vol. 3, pp 137-144.

[29] Chen, A., Lu, J., Lü, J. \& Yu, S. (2006) "Generating hyperchaotic Lü attractor via state feedback control," Physica A, Vol. 364, pp 103-110.

[30] Hahn, W. (1967) The Stability of Motion, Springer, New York.

\section{Authors}

Dr. V. Sundarapandian earned his Doctor of Science degree in Electrical and Systems Engineering from Washington University, Saint Louis, USA in 1996. He is a Professor at the Research and Development Centre, Vel Tech Dr. RR \& Dr. SR Technical University, Chennai, Tamil Nadu, India. He has published over 260 refereed papers in international journals. He has published over 100 papers in National Conferences and over 60 papers in International Conferences. He is the Editor-in-Chief of the AIRCC Journals - International Journal of Instrumentation and Control Systems, International Journal of Control Systems and Computer Modelling, and International Journal of Information Technology, Control and Automation. His research interests are Linear and Nonlinear Control Systems, Chaos Theory and Control, Soft Computing, Optimal Control, Process Control, Operations Research, Mathematical Modelling, Scientific Computing using MATLAB and MATLAB.

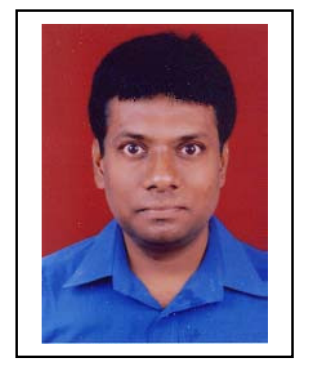

